\title{
EVOLUTION OF SONG CULTURE IN THE ZEBRA FINCH
}

\author{
OLGA FEHER \\ Biology Department, The City College of New York \\ $138^{\text {th }}$ St. at Convent Ave. New York, NY, 10031 USA \\ PARTHA P. MITRA \\ Cold Spring Harbor Laboratory, Cold Spring Harbor, USA \\ KAZUTOSHI SASAHARA \\ Laboratory for Biolinguistics, RIKEN Brain Science Institute \\ 2-1 Hirosawa, Wako-shi, Saitama, 351-0198, Japan \\ OFER TCHERNICHOVSKI \\ Biology Department, The City College of New York \\ $138^{\text {th }}$ St. at Convent Ave. New York, NY, 10031 USA
}

\begin{abstract}
Similar to humans, juvenile songbirds learn their vocal repertoire by imitating adult individuals. When raised in social and acoustic isolation, birds can still sing, but they produce a highly abnormal song. What happens when such an abnormal song is culturally transmitted over generations? To examine this question we placed an isolate (non-tutored) adult bird in a large sound box together with females (who do not sing) and allowed them to breed for a few generations, while recording audio and video so as to track the social interactions and singing behavior. We found that the juveniles readily imitated the isolate song of their father, and yet, small but systematic variations in vocal performance accumulated over generations such that the third generation of learners already sang normal zebra finch song. Here we investigate this cultural evolution process.

Although birdsong is fundamentally different from human language, there are some parallels between early human speech acquisition and the song development of birds (Doupe \& Kuhl, 1999). For example, just as human speech develops hierarchically from phonemes to words and then sentences via babbling, birdsong develops similarly from notes that make up motifs that, in turn, make up bouts. In addition, in both humans and songbirds innate biases and constraints interact with environmental influences to produce the final vocal output. These factors may give rise to complex communication. In many birdsong species, the songs exhibit "dialects" that vary geographically, and females prefer males who sing familiar dialects (Clayton, 1998). Strikingly, on a large geographical scale, there is a convergence of dialects within a species. This effect, called song universals, was qualitatively documented by Marler and Tamura (1962), and it is thought to result from innate perceptual biases.
\end{abstract}


Our experiment is akin to creolization, a process whereby children who learn a pidgin language (a simple improvised language characterized by rudimentary grammar and a small vocabulary) as a first language change it so that it becomes a complex natural language within a few generations. However, our colony was monitored closely, and we were able to observe social interactions that might drive cultural evolution from moment to moment and across generations. We were able to plot developmental trajectories and study the gradual vocal changes made to the newly emerging, complex song, under experimental control.

The vocal output of the isolated colony was recorded continuously and the adult songs were compared to the original founder's and other learners' songs. Although the only available model in our colony was highly abnormal, the young birds readily imitated it. Most of our birds sang songs that resembled most closely their father's song, but when there were male siblings in a clutch, their songs tended to diverge (Tchernichovski \& Nottebohm, 1998) and resulted in greater differences from the father's and the founder's song. The differences were either deviation in acoustic structure from the tutor syllables or (more rarely) the emergence of new syllable types. When young birds added novel syllable types to their song, other juveniles incorporated these into their songs. More interestingly, the changes in acoustic structure appeared to be directional and gradual, when observed over generations. For example, abnormal features of the isolate syllables (e.g. very long durations) turned gradually more normal (shorter) over generations. Within three generations, wide-band, noisy syllables and abnormally long, frequency modulated harmonics were eliminated or changed acoustically to resemble features of normal song. With every group of young learners this trend continued. By the seventh clutch, the song was indistinguishable from normal zebra finch song. We repeated the experiment in an impoverished social setting, where we trained young birds by nontutored adults one on one, and then used them to tutor juveniles and so on. We have attained similar results. In the first generation, there were immediate changes made to the model song, and these changes became more amplified with new generations of learners. Thus, complex social interactions were not necessary to jumpstart cultural evolution, but they may have influenced its speed. In this paradigm, we can observe cultural evolution in real time, and see what innate and social biases act on the evolution and preservation of a local dialect. The biological processes we observed parallel Kirby's iterative learning model for the evolution of language (Smith et al., 2003), in which an individual's competence in producing language depends on his observations of another individual, and cultural transmission results in increased complexity.

\section{References}

Doupe, A.J. and Kuhl, P.K. (1999) Birdsong and Human Speech: Common Themes and Mechanisms. Annu. Rev. Neurosci., 13, 567-631.

Clayton, N.S. (1988) Song discrimination learning in zebra finches. Anim. Behav., 31, 1061-1024.

Marler P. and M. Tamura. 1962. Song "dialects" in three populations of White-crowned Sparrows. Condor., 64, 368-377.

Smith, K., Kirby, S., and Brighton, H. (2003). Iterated learning: a framework for the emergence of language. Artificial Life, 9(4), 371-386. 
Tchernichovski, O. and Nottebohm, F. (1998). Social inhibition of song imitation among sibling male zebra finches. PNAS, 95(15), 8951-8956. 